\title{
EFICÁCIA DO ALONGAMENTO MANTER- RELAXAR NO GANHO DE AMPLITUDE DE MOVIMENTO ARTICULAR DO OMBRO
}

\author{
EFFECTIVENESS OF THE KEEP-RELAX ELONGATION \\ IN THE GAIN OF JOINT RANGE OF MOTION OF THE SHOULDER
}

\begin{abstract}
Joel Freires de Alencar Arrais*a, Dálet da Silva Nascimentoa, Janaína Oliveira de Menezesa, Marina Rodrigues da Silva Batistaa, Diogo Caldeira Ramos ${ }^{b}$, Rodrigo Luis Mousinho Gomes
\end{abstract}

Faculdade de Medicina Estácio de Juazeiro do Nortea Faculdade Inspirarb Universidade Federal de Pernambucoc E-mail: joel.freires00@gmail.com

\section{RESUMO}

A Facilitação Neuromuscular Proprioceptiva (FNP) é um método que abrange inúmeras técnicas utilizadas diariamente em nossa prática clínica. O alongamento manter-relaxar e as diagonais do método são umas delas e possuem uma vasta literatura mostrando a eficácia no ganho de movimento articular ou na neurologia. Desta maneira, este estudo objetiva avaliar os efeitos imediatos do alongamento Manter-Relaxar (MR) do FNP associado as diagonais do membro superior. Trata-se de um estudo quase-experimental, realizado na Faculdade Estácio de Juazeiro do Norte com 30 indivíduos acadêmicos da referida instituição de 20 a 30 anos de idade. Em que o protocolo de alongamento deu-se através do alongamento manter e relaxar associado com as diagonais do membro superior. A análise foi realizada no programa SPSS versão 23, adotando como significância valor de $p \leq 0,05$. 0 estudo foi composto por $20(66,66 \%)$ do sexo feminino e $10(33,34 \%)$ do sexo masculino com idade média de $22,41 \pm 2,19$ anos. Houve diferença significativa entre os valores de amplitude antes e após a técnica MR em todos os movimentos avaliados, com valores de $p<0,05$ para os movimentos de flexão e abdução, em ambos os membros. A técnica MR associada as diagonais do FNP é uma excelente terapêutica para o ganho imediato de amplitude de movimento articular, o que caracteriza o alongamento de um maior grupo muscular simultaneamente.

Palavras-chave: FNP; Exercício de alongamento muscular; Membro superior.

\section{ABSTRACT}

Proprioceptive Neuromuscular Facilitation (PNF) is a method that encompasses numerous techniques used daily in our clinical practice. The keep-relax stretching and the method's diagonals are one of them, and have a vast literature showing efficacy in joint motion gain or neurology. Thus, this study aims to evaluate the immediate effects of the keep-relax stretching (MR) of PNF associated to the diagonals of the upper limb. This is a quasi-experimental study, carried out at the Estácio of Juazeiro of Norte Medical School, with 30 students aged 20 to 30 years old. The stretching protocol was done through the keep and relax stretching associated with the diagonals of the upper limb. The analysis was performed in the SPSS program version 23, adopting as significance value $p \leq 0.05$. The study was composed of 20 (66.66\% female and 10 (33.34\%) male subjects with a mean age of $22.41 \pm 2.19$ years. There was a significant difference between amplitude values before and after the MR technique in all movements evaluated, with $p$ values of $<0.05$ for flexion and abduction movements, in both limbs. The MR technique associated with FNP diagonals is an excellent therapy for the immediate gain of joint range of motion, which characterizes the stretching of a larger muscle group simultaneously.

Keyword: PNF; Muscle Stretching Exercises; Upper Extremity. 


\section{V.10 N.1 (2022) ISSN: 2317-434X}

\section{INTRODUÇÃO}

O ombro ou cíngulo do membro superior é a região mais complexas do corpo humano, possuindo movimentos nos três planos corporais (sagital, lateral e transversal). É composto por cinco articulações: glenoumeral (com 3 graus de liberdade), esternoclavicular, acromioclavicular, coracoclavicular e escapulotorácica (CORREIA, 2011; MARIEB, WILHELM; MALLATT, 2014; PORTELA, 2016).

Os músculos dessa região apresentam função ativa quando relacionado a geração de força para a realizar as atividades diárias e função passiva quando considerado a estabilização do membro superior ao tronco. Alguns músculos (mm.) do membro superior denominados de toracoapendiculares tem seus pontos de fixação na região cervical, dorso e abdome, os quais estão sobrepostos à caixa torácica, como exemplo: $\mathrm{mm}$. peitoral menor, peitoral maior, subescapular e serrátil anterior (CORREIA, 2011; MOORE; DALLEY; AGUR, 2011).

A Facilitação Neuromuscular Proprioceptiva (FNP) é um conceito antigo criado nos anos 40, utilizado para diversos fins terapêuticos, é constituído por alguns tipos de alongamento muscular, a técnica Manter-Relaxar (MR) é uma delas. O alongamento MR possui efetividade no ganho de amplitude de movimento articular (ADM), é realizado no músculo que limita o movimento articular, utiliza da combinação de contrações isométricas dos mm. gerando uma inibição autogênica (relaxamento) das fibras musculares (ADLER; BECKERS; BUCK, 2007; KAY; DODS; BLAZEVICH, 2016; KISNER; COLBY, 2016; MORCELLI; OLIVEIRA; NAVEGA, 2013; SILVA et al., 2010).

As diagonais do FNP do membro superior envolvem a soma de movimentos que acontecem simultaneamente. A Diagonal Primitiva Flexora inclui os movimentos de flexão, abdução e rotação externa da art. Glenoumeral, ativando maiores números de fibras musculares da região do ombro, cervical e caixa torácica, ativando como agonistas os $\mathrm{mm}$. trapézio, elevador da escápula, serrátil anterior, deltoide fibras superiores e redondo menor. Já a Diagonal Primitiva Extensora realiza os movimentos de extensão, adução e rotação interna, ativando como agonistas os $\mathrm{mm}$. serrátil anterior, peitoral menor e maior, romboides, redondo maior e subescapular (ADLER; BECKERS; BUCK, 2007; MORENO; SILVA; GONÇALVES, 2005; YOUDAS et al., 2012). Ao trabalhar com contrações dos $\mathrm{mm}$ da diagonal extensora, consequentemente, alonga-se os $\mathrm{mm}$ da diagonal flexora a qual produz os movimentos avaliados na conduta do presente estudo.

Diante o exposto, acredita-se que a associação de duas técnicas do FNP (alongamento MR e diagonal primitiva flexora e extensora) seja capaz de gerar resultados no ganho de ADM, já que a diagonal ativa um maior número de $\mathrm{mm}$., sendo assim, alongando também maiores números de fibras musculares. Com isso, este estudo objetiva avaliar os efeitos imediatos da associação das técnicas do FNP na ADM do ombro para os movimentos de flexão, abdução e rotação externa.

\section{METODOLOGIA}

Trata-se de um estudo quase-experimental, exploratório-descritivo de abordagem quantitativa. É resultado de uma segunda pesquisa a qual visava avaliar os efeitos do mesmo alongamento no sistema respiratório e que respeitou todas as normas para desenvolvimento da mesma, sendo aprovado pelo Comitê de Ética e Pesquisa da Faculdade de Medicina Estácio de Juazeiro do Norte através do registro $\mathrm{N}^{\circ} 3.266 .473$.

O presente estudo tem como critérios de inclusão: acadêmicos da referida instituição de 20 a 30 anos de idades, ambos os gêneros e identidade de gênero, com redução na amplitude de movimento do ombro para os movimentos de flexão, abdução e rotação externa avaliados no dia da coleta de dados. Como critérios de exclusão: possuir alguma doença ou histórico de cirurgia no ombro, fraturas na cintura escapular, ter realizado qualquer tipo de exercício no membro superior antes da coleta de dados.

A coleta de dados deu-se entre os meses de abril e maio de 2019 na Clínica Escola de Fisioterapia da Instituição supracitada. A triagem dos participantes era realizada através de uma anamnese contendo informações pessoais, dados sobre doenças passadas e exame físico através da goniômetria dos movimentos de flexão e abdução com o indivíduo em ortostatismo e rotação externa com o mesmo deitado em decúbito dorsal em ambos os braços, em que estariam aptos a participarem do estudo os indivíduos que apresentarem redução do ADM na avaliação, para então serem realizados os alongamentos e reavaliados após a conduta. Durante a coleta de dados foram avaliadas 48 pessoas, sendo que através dos critérios citados anteriormente foram incluídos 30 indivíduos.

A goniômetria é realizada com finalidade de medir os ângulos articulares presentes nas articulações humanas, a partir do goniômetro universal. Podem ser de material plástico ou metálico com diferentes tamanhos. Contendo um corpo e dois braços: um móvel e outro fixo, que devem estar posicionados na hora da avaliação de forma que o braço móvel possa acompanhar o movimento até ADM total. São observados no corpo escalas com um 


\section{V.10 N.1 (2022) ISSN: 2317-434X}

giro completo de $360^{\circ}\left(0\right.$ a $\left.360^{\circ}\right)$ ou meio giro com apenas $180^{\circ}\left(0\right.$ a $\left.180^{\circ}\right)$. É um material barato, de fácil manuseio devido a seu tamanho que permite que as medidas sejam tomadas rapidamente (RODRIGUES-OLIVEIRA et al.,
2016; MARQUES, 2014). Para análise da goniômetria no presente estudo foi utilizado os parâmetros do Manual de goniômetria, $3^{a}$ edição (MARQUES, 2014).

Figura 1. Valores de referência para gonometria de ombro.

\begin{tabular}{|c|c|c|}
\hline ARTICULAÇÃo & MOVIMENTO & GRAUS DE MOVIMENTO \\
\hline Ombro & Flexão & 0 a 180 \\
& Extensão & 0 a 45 \\
& Adução & 0 a 40 \\
& Abdução & o a 180 \\
& Rotação medial & a 90 \\
& Rotação lateral & a 90 \\
\hline
\end{tabular}

Fonte: MARQUES (2014).

Em um único dia, foi realizada a avaliação, intervenção e logo após a reavaliação de cada indivíduo. $\mathrm{O}$ alongamento foi realizado associado com as diagonais do método FNP do membro superior. Com o indivíduo em decúbito dorsal com um membro superior para fora da maca, o braço elevado passivamente em direção a diagonal primitiva flexora realizando os movimentos de flexão, abdução e rotação externa até que fosse relatado desconforto leve de estiramento muscular, em seguida realizava-se a contração isométrica de 5 segundos em direção ao retorno do movimento inicial caracterizando os movimentos de extensão, adução e rotação interna (diagonal primitiva extensora), finalizasse com o alongamento passivo da musculatura que realizou a isometria forçando no limite do movimento por 15 segundos com o indivíduo referindo desconforto leve de estiramento muscular. O protocolo foi repetido duas vezes em cada membro.

A análise de dados foi desenvolvida no programa SPSS, versão 23, a partir do qual foram gerados resultados descritos de média e desvio padrão, bem como verificada a significância estatística por meio do teste t para amostras pareadas (antes e depois da intervenção), adotando como critério de significância um valor de $\mathrm{p}<0,05$.

\section{RESULTADOS}

O estudo foi composto por uma amostra de 30 indivíduos, dos quais, $33,34 \%$ eram do sexo masculino e $66,66 \%$ do sexo feminino. A média de idade da amostra foi de $22,43 \pm 2,19$. Na tabela 1 identifica-se valores amostrais e média de idade por sexo. Na primeira avaliação para quantificar o movimento da articulação glenoumeral, foram encontradas reduções principalmente na flexão e abdução, em comparação com a rotação externa que obteve menor índice de redução. No gráfico 1 observa-se a média de ADM antes e após no protocolo de alongamento no membro superior direito.

Tabela 1. Desenho da amostra

\begin{tabular}{ccc}
\hline & Grupo Experimento & Idade \\
\hline Experimento & $\mathrm{N}=30$ & $22,41 \pm 2,19$ \\
Mulheres & $\mathrm{N}=20(66,66 \%)$ & $22,55 \pm 2,38$ \\
Homens & $\mathrm{N}=10(33,34 \%)$ & $22,20 \pm 1,60$ \\
\hline
\end{tabular}

Fonte: (Autoral, 2019).

Gráfico 1. Valores de comparação da goniometria dos movimentos do ombro direito antes e após o alongamento 


\section{V.10 N.1 (2022) ISSN: 2317-434X}

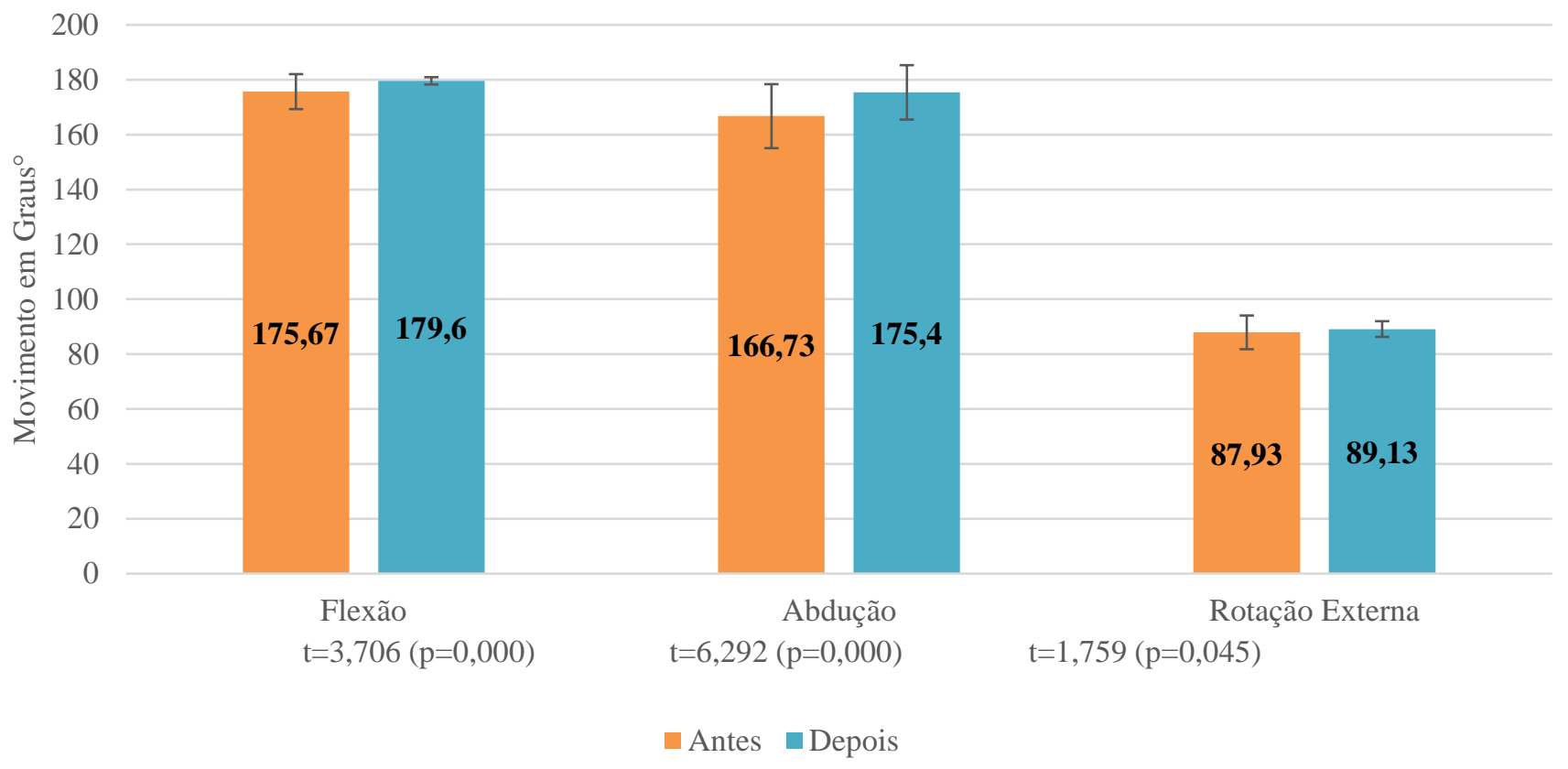

Fonte: (Autoral, 2019).

Pode-se observar no gráfico que na articulação glenoumeral direita houve ganho expressivo nos três movimentos avaliados sendo que, houve maior ganho nos movimentos de abdução $\left(+8,67^{\circ}\right)$ e flexão $\left(+3,93^{\circ}\right)$, seguido do movimento de rotação externa $\left(+1,20^{\circ}\right)$ comparando com o valor de goniometria inicial com a avaliação realizada após a técnica e evidenciado também pelo valor de $\mathrm{p}$ menor que 0,001 nos dois primeiros movimentos. Já no gráfico 2 observa-se a média de ADM antes e após o protocolo de alongamento no membro superior esquerdo.

Gráfico 2. Valores de comparação da goniômetria dos movimentos do ombro esquerdo antes e após o alongamento.

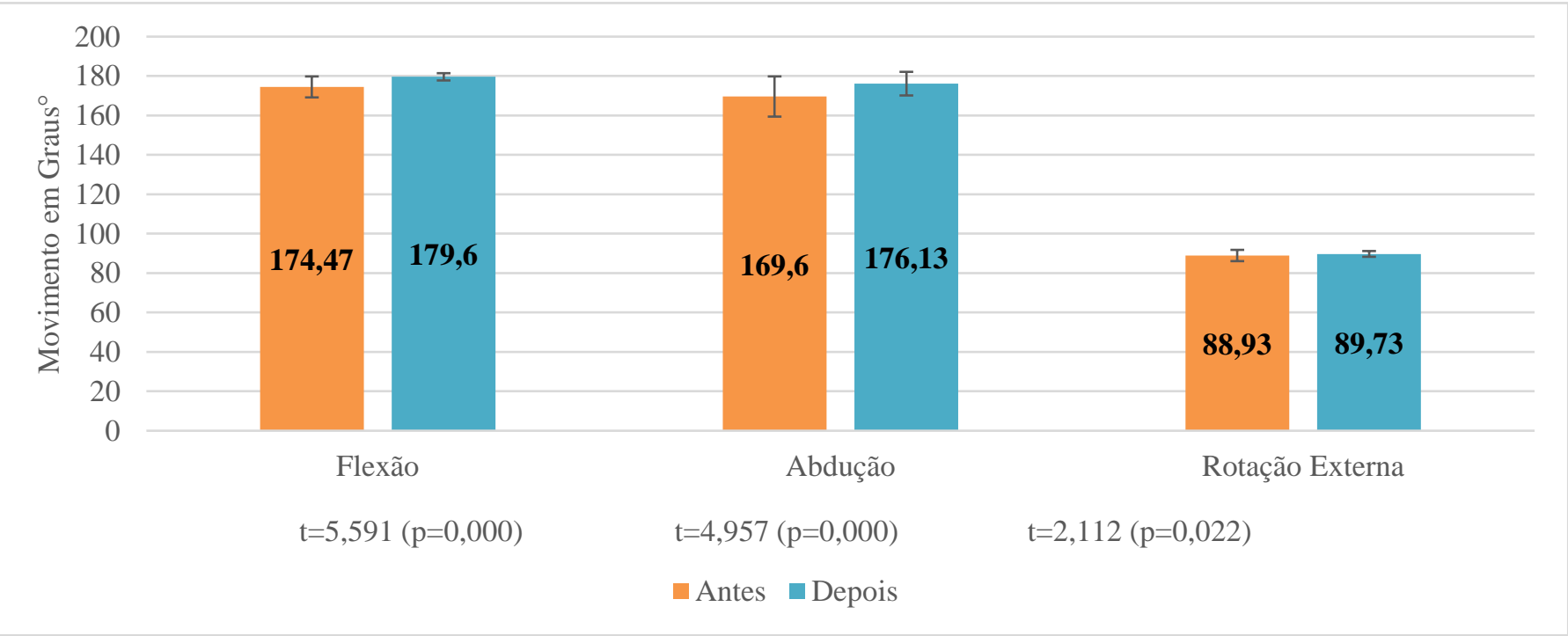

Fonte: (Autoral, 2019).

Com os dados expostos observa-se que na articulação glenoumeral esquerda aconteceu o mesmo que 


\section{V.10 N.1 (2022) ISSN: 2317-434X}

na articulação glenoumeral direita, tanto em relação ao ganho de ADM, tendo em vista que todos os movimentos avaliados melhoraram após a técnica de alongamento, quanto nos movimentos que apresentaram maior ganho: abdução $\left(+6,53^{\circ}\right)$, flexão $\left(+5,13^{\circ}\right)$ e rotação externa $(+$ $\left.0,80^{\circ}\right)$.

Confirmado através dos cálculos estatísticos, que observou o crescimento significativo após a técnica MR associada a diagonal em todos os movimentos avaliados, e obteve valor de $\mathrm{p}<0,01$ para flexão e abdução e valor de $\mathrm{p}<0,05$ para rotação externa.

\section{DISCUSSÃO}

Para o American College of Sports Medicine (2014), os exercícios de alongamento muscular demonstram em todas as faixas etárias melhora na amplitude e flexibilidade articular, obtendo resposta imediata na ADM, apresentando também respostas tardias de manutenção da ADM ganha. A prática regular resulta na redução de lesões musculoesqueléticas, prevenção de dores ou um retardo no início das dores musculares. Já a flexibilidade diminuída, é associada a limitações funcionais levando ao comprometimento da saúde do indivíduo, consequentemente, influenciando na qualidade de vida, pois os alongamentos musculares são capazes também de reduzir o estresse ocupacional do trabalho (BRITO et al., 2016; CÉSAR et al., 2018; COSTA et al., 2017).

Os alongamentos possuem diversos efeitos positivos para o ser humano, e esses efeitos são observados em todas as faixas etárias. O principal efeito é o ganho imediato de ADM e flexibilidade articular, além da preservação desse ganho por determinado período de tempo sem prejuízo na força muscular. Sua prática regular possibilita ainda a prevenção de lesões e dores musculoesqueléticas (AMERICAN COLLEGE OF SPORTS MEDICINE, 2014; BRITO et al., 2016; CÉSAR et al., 2018). O FNP é um método que visa melhorar a funcionalidade do indivíduo seja por estímulos de facilitação, inibitórios, estímulos de fortalecimento ou relaxamento muscular. Tais exercícios visam primordialmente o ganho de adm (ABREU et al., 2015; TUCKER; SLONE, 2016).

A técnica MR é uma das técnicas da FNP que possuem efetividade no ganho imediato da flexibilidade (MORCELLI; OLIVEIRA; NAVEGA, 2013). Que utiliza a combinação de contrações da musculatura com o alongamento proporcionando a inibição autogênica do músculo onde se buscam trabalhar o estiramento muscular, é necessário o auxílio de um colega ou terapeuta para realização da contração na posição de alongamento máximo pois podem ser dolorosas e induzindo a danos musculares (KAY; DODS; BLAZEVICH, 2016; SILVA et al., 2010).

Alguns estudos já compararam e comprovaram que as técnicas do FNP apresentam maior resultado no ganho de ADM (MORCELLI; OLIVEIRA; NAVEGA, 2013; ROSA et al., 2006; SILVA; PIN; SILVA FILHO, 2015). O resultado foi possível, pois após a contração isométrica das fibras musculares ocorre a inibição autogênica que inibe os órgãos tendinosos, causando o relaxamento do músculo (ADLER; BECKERS; BUCK, 2007; GAMA et al., 2007).

Corroborando com o presente estudo que observou após o uso da técnica MR associado com as diagonais do FNP houve ganho das ADM do ombro. Tal resultado acontece, pois ocorre um relaxamento prévio da fibra muscular que estão limitando os movimentos, explicada por Alder, Beckers e Buck (2007) e Gama et al., (2007), que a contração isométrica antes do estiramento causa uma inibição dos órgãos tendinosos, sendo responsável pelo relaxamento muscular.

Também foi observado que o alongamento foi benéfico na diagonal do FNP possivelmente pela maior ativação muscular, melhorando assim a adm. Rosa et al., (2006), assim como Silva, Pin e Silva Filho (2015), comprovam e comparam as técnicas do FNP com outras técnicas de alongamentos musculares, constatando que as técnicas de alongamento do FNP são as que apresentam maior resultado no ganho de ADM, sendo positivo também no ganho imediato de amplitude (MORCELLI; OLIVEIRA; NAVEGA, 2013; SANTOS et al., 2017). O que também foi evidenciado em uma revisão sistemática com meta-análise que as técnicas de FNP quando realizadas junto as diagonais de membros superiores são eficazes no aumento da adm em indivíduos com capsulite adesiva (TEDLA; SANGADALA, 2019).

\section{CONCLUSÃO}

Os resultados obtidos neste estudo, nas condições utilizadas é possível concluir então que o protocolo de FNP utilizado é um programa de exercício eficiente para ganho imediato na ADM do ombro para os movimentos de flexão, abdução e rotação externa. Apesar dos efeitos terem sido significativos nos três movimentos, acredita-se que o protocolo utilizado possua efeitos também em indivíduos com patologias no ombro. Contudo, há a necessidade de avaliação dessa hipótese, além de avaliar a duração que os ganhos se mantiveram nesses indivíduos. 


\section{V.10 N.1 (2022) ISSN: 2317-434X}

\section{REFERÊNCIAS}

ABREU, R. et al. Force irradiation effects during upper limb diagonal exercises on contralateral muscle activation, Journal of Electromyography and Kinesiology, v. 25, n. 2, p. 292-297, 2015.

ADLER, S. S.; BECKERS, D.; BUCK, M. PNF Facilitação Neuromuscular Proprioceptiva: Um guia ilustrado. 2. ed. Barueri, SP: Manole, 2007.

AMERICAN COLLEGE OF SPORTS MEDICINE. Diretrizes do ACSM para os testes de esforço e sua prescrição. 9. ed. Rio de Janeiro: Guanabara, 2014. p 132. Disponível em: < https://www.passeidireto.com/arquivo/30557043/diretrizes-doacsm-para-os-testes-de-esforco-e-sua-prescricao-9-ed-2014 Acesso em: 23 de junho de 2018.

BRITO, E. et al. Flexibilidade, imagem corporal e índice de massa corporal de idosas praticantes de alongamento no centro desportivo municipal (CDM) Santa Maria/2015. Salusvita, v. 35, n. 4, p. 447-487, 2016.

CÉSAR, E. P. et al. Comparação de dois protocolos de alongamento para amplitude de movimento e força dinâmica. Revista Brasileira de Medicina do Esporte, v. 24, n. 1, 2018.

CORREIA, N. A. Estudo da Biomecânica do Ombro. Orientador: Renato Manuel Natal Jorge. 2011. 134 f. Dissertação (Mestrado Integrado em Engenharia Mecânica) Faculdade de Engenharia da Universidade do Porto, Portugal, 2011. Disponível em: file:///D:/Joelson/Downloads/000148631.pdf. Acesso em: 20 jan. 2021.

COSTA, M. V. C. et al. Exercícios de alongamento muscular na percepção de estresse em profissionais de enfermagem: estudo clínico randomizado. Caderno Brasileiro de Terapia Ocupacional, v. 27, n. 2, p. 357-366, 2017.

GAMA, Z. A. S. et al. Influência da frequência de alongamento utilizando facilitação neuromuscular proprioceptiva na flexibilidade dos músculos isquiotibiais. Revista Brasileira de Medicina do Esporte, v. 13, n. 1, p. 33-38, 2007.

KAY, A. D.; DODS, S.; BLAZEVICH, A. J. Anthony. Acute effects of contract-relax (CR) stretch versus a modified CR technique. European Journal of Applied Physiology, v. 116, n. 3, p. 611-621, 2016.

KISNER, C.; COLBY, A. L. Exercícios terapêuticos: Fundamentos e técnicas. Tradução: Lilia Breternitz Ribeiro. 6 ed. Barueri, São Paulo: Manole, 2016.

MARIEB, E. N.; WILHELM, P. B.; MALlATT, J. Anatomia humana. 7. ed. São Paulo: Pearson Education do Brasil, 2014.
MARQUES, A. P. Manual de goniometria, 3. ed. Barueri, São Paulo: Manole, 2014.

MOORE, K. L.; DALLEY, A. F.; AGUR, A. M. R. Anatomia orientada para a clínica. 6. ed. Rio de Janeiro: Guanabara Koogan, 2011.

MORCELLI, M. H.; OLIVEIRA, J. M. C. A.; NAVEGA, M. T. Comparação do alongamento estático, balístico e contrairrelaxar nos músculos isquiotibiais. Fisioterapia e Pesquisa, v. 20, n. 3, p. 244-249, 2013.

MORENO, M. A.; SILVA, E.; GONÇALVES, M. O efeito das técnicas de Facilitação Neuromuscular Proprioceptiva - Método Kabat - nas pressões respiratórias máximas. Fisioterapia em Movimento, v. 18, n. 2, p. 53-61, 2005.

PORTELA, J. P. Cinesiologia. 1. ed. Sobral: Inta, 2016.

RODRIGUES-OLIVEIRA, H. K. et al. Benefício da facilitação neuromuscular proprioceptiva em mulheres mastectomizadas: um estudo piloto. Revista Inova Saúde, v. 5, n. 2, p. 1-15, 2016.

ROSA, A. S. et al. Estudo comparativo entre três formas de alongamento: ativo, passivo e facilitação neuroproprioceptiva. Terapia Manual, v. 4, n. 16, p. 97-101, 2006.

SANTOS, D. et al. Efeito agudo de diferentes técnicas de alongamento na flexibilidade de isquiostibiais. Fisioterapia Brasil, v. 18, n. 6, p. 708-718, 2017.

SILVA, S. A. et al. O efeito do tempo de duas diferentes técnicas de alongamento na amplitude de movimento. ConScientiae Saúde, v. 9, n. 1, p. 71-79, 2010.

SILVA, E. B.; PIN, A. S.; SILVA FILHO, M. Changes in muscle strength in elderly women after proprioceptive neuromuscular facilitation based training. Fisioterapia e Movimento. v. 23, n. 2, p. 357-363, 2015.

TEDLA, J. S.; SANGADALA, D. R. Proprioceptive neuromuscular facilitation techniques in adhesive capsulitis: a systematic review and meta-analysis. Journal of Musculoskeletal and Neuronal Interactions, v. 19, n. 4, p. 482-491, 2019.

TUCKER, W. S.; SLONE, S. W. The acute effects of hold-relax Proprioceptive Neuromuscular Facilitation with vibration therapy on glenohumeral internal-rotation deficit. Journal of Sport Rehabilitation, v. 25, n. 3, p. 248-254, 2016. 


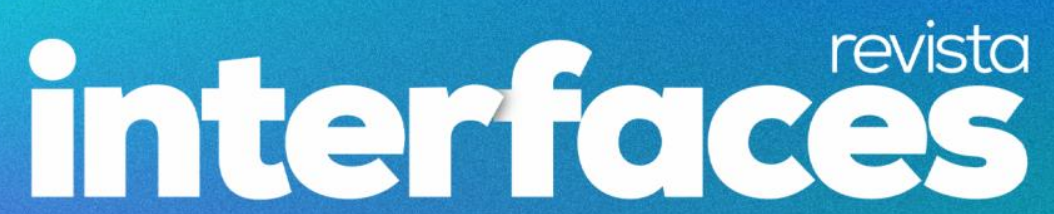

\section{Artigos \\ Originais}

\section{V.10 N.1 (2022) ISSN: 2317-434X}

YOUDAS, J. W. et al. Comparison of muscle activation levels during arm abduction in the plane of the scapula vs. Proprioceptive neuromuscular facilitation upper extremity patterns. Journal of Strength and Conditioning Research. v. 26, n. 4, p. 1058-1065, 2012. 